Case Report

\title{
Long-Lasting Symptomatic Cerebral Hyperperfusion Syndrome following Superficial Temporal Artery-Middle Cerebral Artery Bypass in a Patient with Stenosis of Middle Cerebral Artery
}

\author{
Shinji Shimato (D), Toshihisa Nishizawa, Takashi Yamanouchi, Takashi Mamiya, \\ Kojiro Ishikawa, and Kyozo Kato \\ Department of Neurosurgery, Kariya Toyota General Hospital, 5-15 Sumiyoshi-cho, Kariya City, Aichi 448-8505, Japan \\ Correspondence should be addressed to Shinji Shimato; shinji.shimato@gmail.com
}

Received 9 May 2018; Revised 16 July 2018; Accepted 1 August 2018; Published 23 September 2018

Academic Editor: Norman S. Litofsky

Copyright (C) 2018 Shinji Shimato et al. This is an open access article distributed under the Creative Commons Attribution License, which permits unrestricted use, distribution, and reproduction in any medium, provided the original work is properly cited.

\begin{abstract}
Cerebral hyperperfusion syndrome (CHPS) is a complication that can occur after cerebral revascularization surgeries such as superficial temporal artery- (STA-) middle cerebral artery (MCA) anastomosis, and it can lead to neurological deteriorations. CHPS is usually temporary and disappears within two weeks. The authors present a case in which speech disturbance due to CHPS lasted unexpectedly long and three months was taken for full recovery. A 40-year-old woman, with a history of medication of quetiapine, dopamine 2 receptor antagonist as an antipsychotics for depression, underwent STA-MCA anastomosis for symptomatic left MCA stenosis. On the second day after surgery, the patient exhibited mild speech disturbance which deteriorated into complete motor aphasia and persisted for one month. SPECT showed the increase of cerebral blood flow (CBF) in left cerebrum, verifying the diagnosis of CHPS. Although CBF increase disappeared one month after surgery, speech disturbance continued for additionally two months with a slow improvement. This case represents a rare clinical course of CHPS. The presumable mechanisms of the prolongation of CHPS are discussed, and the medication of quetiapine might be one possible cause by its effect on cerebral vessels as dopamine 2 receptor antagonist, posing the caution against antipsychotics in cerebrovascular surgeries.
\end{abstract}

\section{Introduction}

Superficial temporal artery (STA) to middle cerebral artery (MCA) anastomosis is a surgical procedure of direct revascularization to improve cerebral blood flow and potentially prevent brain infarction for patients with stenoocclusive cerebrovascular diseases [1]. While this surgical procedure has the advantage of rapid improvement of impaired cerebral blood flow $(\mathrm{CBF})$, there is a potential risk for postoperative cerebral hyperperfusion syndrome (CHPS) $[2,3]$.

Cerebral hyperperfusion state is defined as a major increase cerebral blood flow (CBF) following surgical repair that is well above the metabolic demands of the brain tissue, and it can be detected using single-photon emission computed tomography (SPECT) [4]. When hyperperfusion reaches symptomatic state, CHPS is characterized by unilateral headache, facial, and ocular pain, seizures, and focal neurological signs $[4,5]$. With regard to the time course of
CHPS, the symptoms usually start during the acute stage after bypass surgery [6-8], and the duration is $1-2$ weeks in most cases $[6,8,9]$.

We report a case of a patient who suffered from speech disturbance for more than three months due to CHPS after the surgery of STA-MCA anastomosis for symptomatic left MCA stenosis. The characteristics of this case and the mechanism of the long duration of CHPS are discussed.

\section{Case Report}

A 39-year-old woman, who had been taking medication of quetiapine as an antipsychotics for depression, experienced mild dysarthria and visited the department of neurology in our hospital. Her symptom was diagnosed as drug-induced lip dyskinesia, which disappeared in a week. Screening head magnetic resonance imaging (MRI) at this time revealed stenosis of the left MCA with no brain parenchymal lesions 


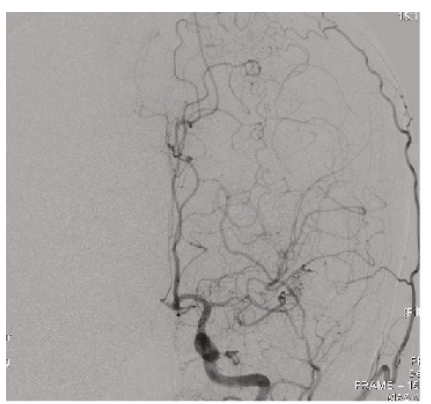

(a)

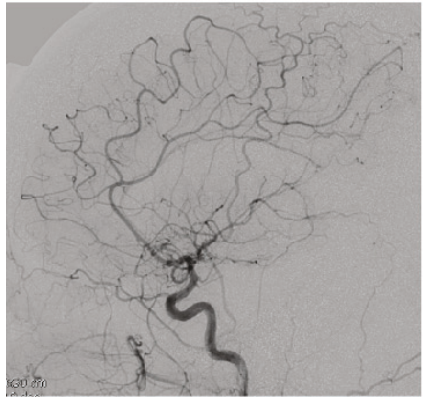

(b)

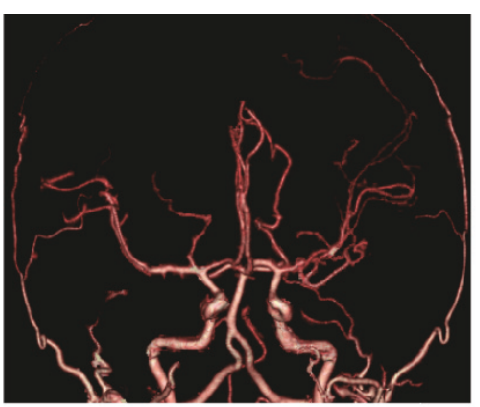

(c)

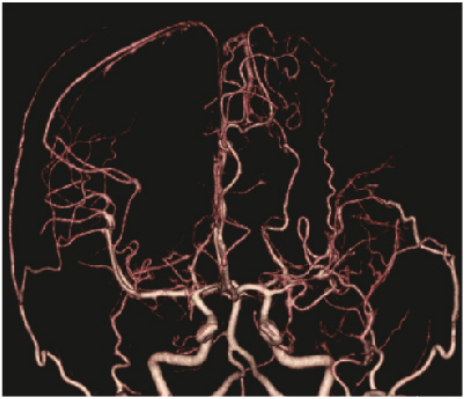

(d)

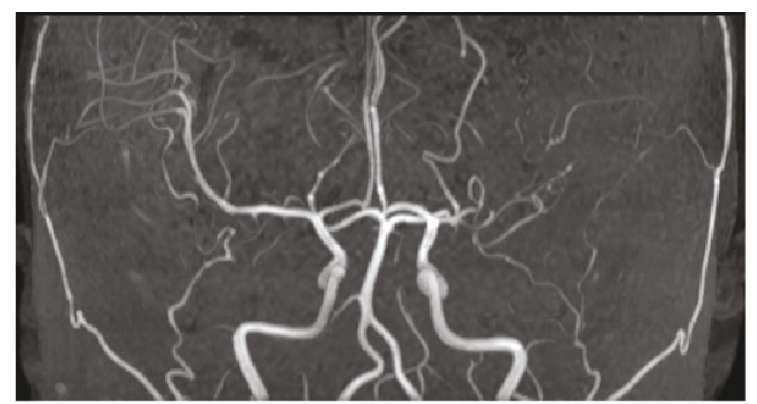

(e)

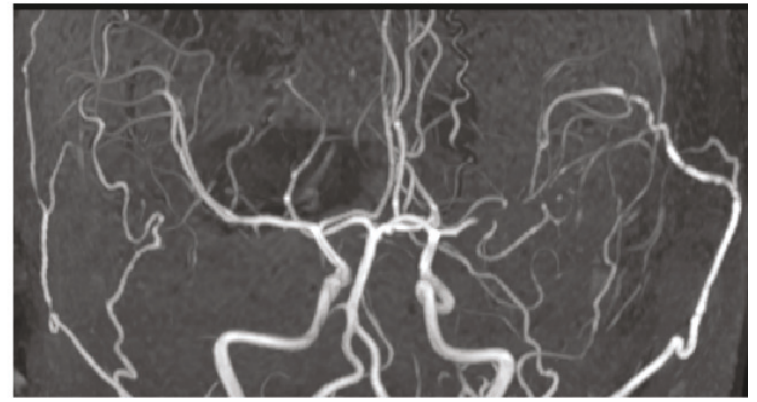

(f)

FIGURE 1: Pre- and postoperative cerebrovascular examinations. (a) and (b) Preoperative digital subtraction angiography showing narrowing in the M1 portion of MCA. (c) Preoperative 3D-CT angiography showing narrowing in the M1 portion of MCA. (d) Postoperative 3D-CT angiography showing the patency of STA-MCA anastomosis. (e) Preoperative magnetic resonance angiography (MRA) showing stenosis of M1 portion of left middle cerebral artery (MCA) and poor visualization of peripheral MCA. (f) Postoperative MRA showing the patency of STA-MCA anastomosis and improvement of blood flow in peripheral MCA.

(Figure 1(e)), why she was consulted to our department. We performed angiography, confirming moderate M1 portion stenosis (Figures 1(a) and 1(b)). SPECT showed no apparent laterality in $\mathrm{CBF}$, thereby we decided to observe her with no treatment.

Seven months later, the patient experienced mild weakness and numbness in her right hand and visited our department. Although MRI showed no apparent ischemic change in her brain, arterial spin labelling (ASL) of MRI detected the decrease of CBF in the left cerebrum (Figure 2(a)), which was thought to well correspond for her symptoms. She was admitted and treated with an antiplatelet agent. Two weeks later, she still complained of numbness in her right hand; thereby, we decided to perform left STA-MCA anastomosis to prevent deterioration of her symptoms. Preoperative SPECT showed no apparent laterality in CBF (Figure 2(c)). On operation, left temporal craniotomy was performed, and the parietal branch of the STA was anastomosed with the M4 portion on the temporal lobe (Figures 1(d) and 1(f)). The intraoperative course was uneventful, and the patient recovered from anesthesia without any new neurological symptoms

Postoperatively, her speech was normal until postoperative day 1 (POD1). On POD2, she exhibited mild speech disturbance, which worsened day by day finally resulting in complete motor aphasia on POD6. Her comprehension was kept normal. On POD3, generalized convulsion occurred, which ceased quickly by diazepam, and levetiracetam was initiated. On the same day, she presented with mild weakness of right upper extremity, which improved gradually and disappeared on POD7. MRI and CT showed no ischemic or hemorrhagic changes, but ASL and SPECT revealed remarkable increase of $\mathrm{CBF}$ in the left cerebrum (Figures 2(b) and 2(d)), by which the symptoms were diagnosed as CHPS. Despite the treatment with strict blood pressure and the administration of edaravone and minocycline, complete motor aphasia remained unchanged on POD21. MRI showed no abnormality except slightly hypointense changes on T2 weighted images and FLAIR (Figure 2(e)). At this point, the patient was discharged partly because of the request from the patient, and we continued to follow her in outpatient visit. One month after the surgery, the patient started to utter words that were not fluent, when SPECT and MRI showed normalization (Figure 2(f)). Thereafter, the improvement of her speech was slow, and totally more than three months was taken for full recovery after the surgery.

\section{Discussion}

Cerebral hyperperfusion syndrome (CHPS) following cerebral revascularization is well recognized particularly in the context of carotid endarterectomy (CEA) and carotid artery stenting (CAS) $[4,10]$. STA-MCA anastomosis, which can be indicated for atherosclerotic diseases as well as moyamoya disease $[1,11]$, is another surgical procedure in which CHPS can occur $[2,3]$. The incidence of postoperative CHPS varies 


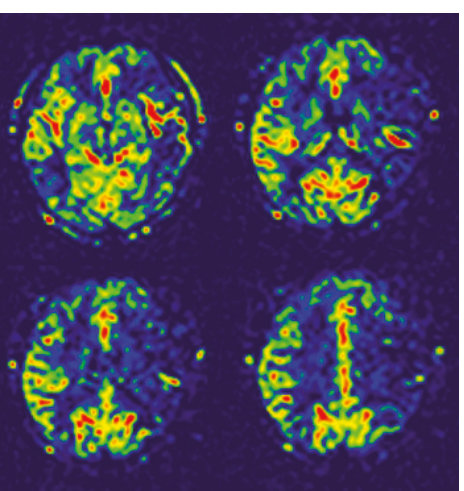

(a)

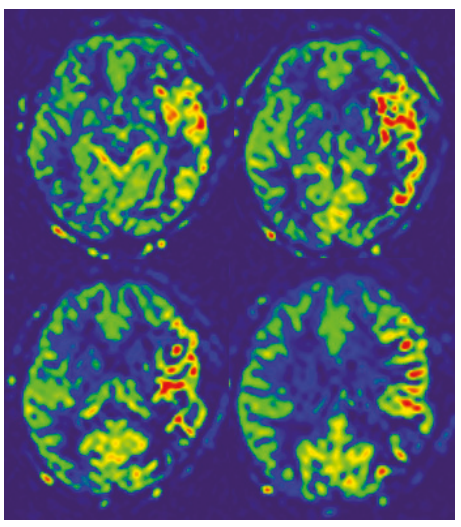

(b)

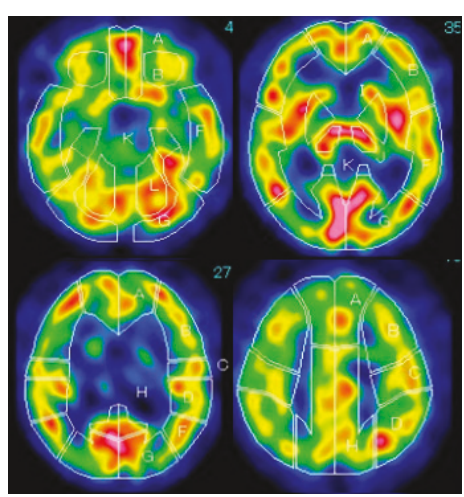

(c)

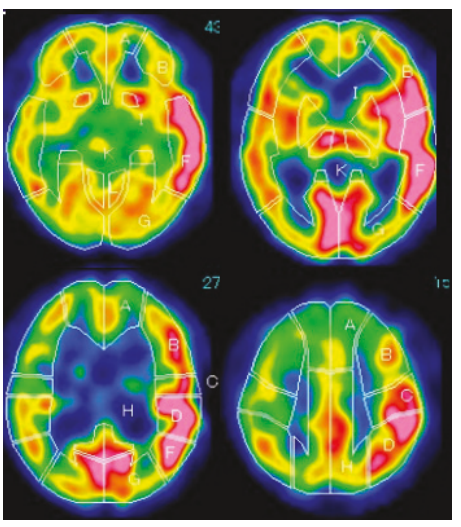

(d)

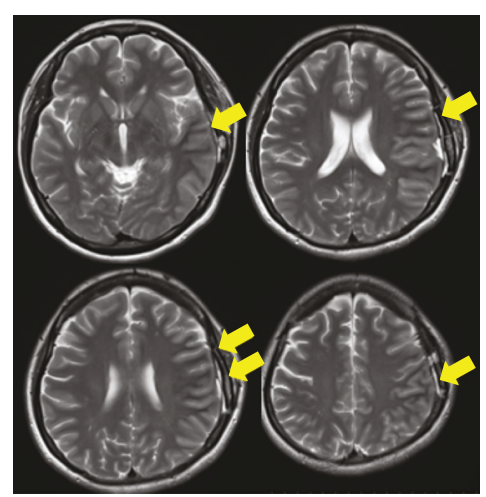

(e)

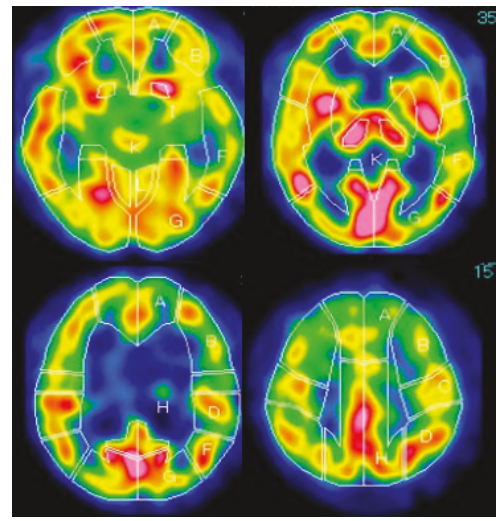

(f)

FIGURE 2: Pre- and postoperative neuroradiologic examinations. (a) Arterial spin labelling (ASL) of MRI showing decrease of CBF in the left brain when the patient presented with right hand weakness and numbness; (b) ASL on postoperative day (POD) 5 showing an increase of CBF in the left cerebrum; (c) preoperative single-photon emission computed tomography (SPECT) showing no apparent laterality in CBF. (d) SPECT on POD12 showing remarkable increase of CBF in the left cerebrum; (e) MRI T2WI on POD16 showing slightly hypointense change in the subcortex of the left cerebrum (arrows). (f) SPECT on POD33 showing normalization of CBF.

according to the surgical procedures or background of the patients, and it has been reported to occur in up to $50 \%$ of patients after STA-MCA anastomosis [2, 3, 10]. Resultant neurological deficits may be permanent and severe if intracranial hemorrhage occurs due to CHPS [12]. Otherwise, hyperperfusion is usually a temporary status that disappears within one week after surgery, and the symptoms are expected to disappear within 1-2 weeks in most cases $[6,8,9]$.

In the present case, the initial presentation of CHPS appears typical in terms of the symptoms as well as the pattern of hyperperfusion on SPECT, because the area of hyperperfusion is frequently in localized brain cortex after STA-MCA anastomosis [6-8], and the focal neurologic signs in accordance with the anatomical location of the site of anastomosis are the most frequent symptoms in STA-MCA bypass surgery [6]. Then, hyperperfusion state detected on ASL or SPECT as well as complete motor aphasia continued for more than three weeks, which was longer than usually seen. More unexpectedly, the improvement of speech was slow even after the normalization of CBF on SPECT, requiring additionally more than two months for full recovery. The cytogenic edema could be a possible cause for persisting neurological deficits in patients with severe hyperperfusion
[13]. In our case, although slightly hypointense change on MRI-T2 and FLAIR images was observed in the subcortex area of the left cerebrum, no changes indicating edema or stroke were seen at all. Epilepsy was denied as well by the fact that no seizures occurred after the initiation of levetiracetam and she was alert all the time. Therefore, we assume that her prolonged symptoms were the sequela of CHPS, and the full recovery of her symptoms could support it.

There are two cases showing long duration of CHPS after STA-MCA bypass reported in the literature, both of which were the patients with moyamoya disease $[6,14]$. In one case by Takemoto et al., a 59-year-old female patient experienced 5 weeks duration of aphasia and right hemiparesis [14], and the period of hyperperfusion detected on SPECT was three weeks, which is similar to that in our case. In another case shown in the study of 27 consecutive patients by Fujimura et al. [6], a 37-year-old male suffered from 30-day duration of aphasia and sensory disturbance. In their case, SPECT was taken only on POD2 and 7, and the actual duration of hyperperfusion on SPECT is unknown. The total duration of symptoms due to CHPS in our case is the longest in the literature, and this is the first case in atherosclerotic diseases, to our knowledge. 
Although underlying mechanisms for hyperperfusion remain undetermined, possible pathophysiology for CHPS has been suggested to be the impaired autoregulation, endothelial dysfunction mediated by free radicals, breakdown of the baroreceptor reflex, and breakdown of bloodbrain-barrier (BBB), which results from a rapid increase in cerebral blood flow $[15,16]$. The impaired autoregulation is thought to be the central mechanism because the reduction of preoperative $\mathrm{CBF}$ and cerebral vascular reserve capacity (CVRC), which lead to impaired autoregulation, are known as the significant risk factors for hyperperfusion $[13,15]$. In the case reported by Takemoto et al. mentioned above, regional differences in the functional recovery of cerebrovascular reactivity were suggested to the possible cause, based on the observation that the area of hyperperfusion shifted from basal ganglia to the cortex surrounding the anastomosis for three weeks [14]. In our case, speech disturbance persisted long while paresis of right upper extremity disappeared on POD7 although relatively large area covering motor area for right upper extremity was affected. Subtle differences between regions in the functional recovery of cerebrovascular reactivity might have led to such discrepancy in the length of the symptoms, which image studies could not detect. Preoperative measurement of $\mathrm{CBF}$ with acetazolamide in addition to that at the resting state is demonstrated to be helpful to predict the development of CHPS [17], and it might have shown some evidences revealing impaired autoregulation with regional differences.

Positron emission tomography (PET) is another tool to detect hyperperfusion in different aspects than MRI or SPECT can detect, and the decrease of oxygen extraction fraction (OEF) and the tendency of high cerebral blood volume (CBV) are reported to be the factors observed on PET in patients who developed CHPS [18]. In particular, prolonged recovery of CBV values is suggested to have a key role in the development of hyperperfusion and the associated clinical symptoms [18]. Therefore, in our case, impaired oxygen metabolism or increased CBV might have persisted long even after the normalization of CBF.

Considering that the symptoms persisted extremely long despite all possible treatments including strict control of blood pressure and the administration of edaravone and minocycline [19-21], there might have been some particular factors in this patient. Here, in view of the daily medication of quetiapine that the patient had been taking for depression, we suggest one hypothesis that quetiapine, which has an antagonistic action on dopamine 2 receptor, might have affected cerebral vessels and prolonged hyperperfusion, based on the previous reports regarding the effect of dopamine 2 receptor antagonist (D2 antagonist) on cerebral vessels [22-24]. Ion et al. reported in a postmortem study for Schizophrenia patients that D2 antagonist may affect the neurovascular unit and small structural microvascular changes [22]. Another study with adult rats by Gepdiremen et al. showed that wall thickness of basilar artery decreased significantly due to vasoconstriction when haloperidol, D2 antagonist, was given [23]. In addition, similar antipsychotics are reported to be likely to incur cytotoxic effects and apoptosis of BBB endothelia with an impairment of barrier functionality [24].
In our case, the cerebral vessels had presumably been exposed to D2 antagonist for a long time; thus, they might have sustained the impairment of vasoconstriction or BBB, leading to prolonged dilatation of the vessels or impaired oxygen metabolism following the increase of CBF after STA-MCA bypass surgery. In this regard, we should mention that we restarted the daily medication of quetiapine immediately after the surgery and continued it throughout the course. The hypothesis mentioned above could not be verified, and no cases with any brain diseases have been reported in which antipsychotics is suggested to affect cerebral blood flow, to our knowledge. Therefore, the degree of the influence of quetiapine on CHPS in this case could not be extrapolated. However, our case with such rare relationship could pose the alarm regarding the management of antipsychotics for cerebral revascularization surgeries.

This case represented a rare clinical course of CHPS. Factors causing long duration could not be determined, but we suggest a possibility of the involvement of antipsychotics by its effect on cerebral vessels as D2 antagonist. Further studies are needed to elucidate the mechanisms of CHPS and to establish the optimal treatment strategy for better prognosis.

\section{Conflicts of Interest}

There are no conflicts of interest about this study.

\section{References}

[1] C. Muroi, N. Khan, D. Bellut, M. Fujioka, and Y. Yonekawa, "Extracranial-intracranial bypass in atherosclerotic cerebrovascular disease: Report of a single centre experience," British Journal of Neurosurgery, vol. 25, no. 3, pp. 357-362, 2011.

[2] J. E. Kim and J. S. Jeon, "An update on the diagnosis and treatment of adult Moyamoya disease taking into consideration controversial issues," Neurological Research, vol. 36, no. 5, pp. 407-416, 2014.

[3] J. W. Hwang, H. M. Yang, H. Lee et al., "Predictive factors of symptomatic cerebral hyperperfusion after superficial temporal artery-middle cerebral artery anastomosis in adult patients with moyamoya disease," British Journal of Anaesthesia, vol. 110, no. 5, pp. 773-779, 2013.

[4] D. G. Piepgras, M. K. Morgan, T. M. Sundt Jr., T. Yanagihara, and L. M. Mussman, "Intracerebral hemorrhage after carotid endarterectomy," Journal of Neurosurgery, vol. 68, no. 4, pp. 532536, 1988.

[5] R. A. Solomon, C. M. Loftus, D. O. Quest, and J. W. Correll, "Incidence and etiology of intracerebral hemorrhage following carotid endarterectomy," Journal of Neurosurgery, vol. 64, no. 1, pp. 29-34, 1986.

[6] M. Fujimura, T. Kaneta, S. Mugikura, H. Shimizu, and T. Tominaga, “Temporary neurologic deterioration due to cerebral hyperperfusion after superficial temporal artery-middle cerebral artery anastomosis in patients with adult-onset moyamoya disease," World Neurosurgery, vol. 67, no. 3, pp. 273-282, 2007.

[7] M. Fujimura, T. Kaneta, H. Shimizu, and T. Tominaga, "Symptomatic hyperperfusion after superficial temporal artery Middle cerebral artery anastomosis in a child with moyamoya 
disease," Child's Nervous System, vol. 23, no. 10, pp. 1195-1198, 2007.

[8] M. Fujimura, H. Shimizu, T. Inoue, S. Mugikura, A. Saito, and T. Tominaga, "Significance of focal cerebral hyperperfusion as a cause of transient neurologic deterioration after extracranial-intracranial bypass for moyamoya disease: Comparative study with non-moyamoya patients using n-isopropylp-[ 123I]iodoamphetamine single-photon emission computed tomography," Neurosurgery, vol. 68, no. 4, pp. 957-965, 2011.

[9] T. Ishikawa, K. Houkin, H. Kamiyama, and H. Abe, "Effects of surgical revascularization on outcome of patients with pediatric moyamoya disease," Stroke, vol. 28, no. 6, pp. 1170-1173, 1997.

[10] K. Ogasawara, N. Sakai, T. Kuroiwa et al., "Intracranial hemorrhage associated with cerebral hyperperfusion syndrome following carotid endarterectomy and carotid artery stenting: retrospective review of 4494 patients," Journal of Neurosurgery, vol. 107, no. 6, pp. 1130-1136, 2007.

[11] S. Miyamoto, "Study Design for a Prospective Randomized Trial of Extracranial-Intracranial Bypass Surgery for Adults with Moyamoya Disease and Hemorrhagic Onset: The Japan Adult Moyamoya Trial Group," Neurologia medico-chirurgica, vol. 44, no. 4, pp. 218-219, 2004.

[12] F. Matano, Y. Murai, T. Mizunari, K. Adachi, S. Kobayashi, and A. Morita, "Intracerebral Hemorrhage Caused by Cerebral Hyperperfusion after Superficial Temporal Artery to Middle Cerebral Artery Bypass for Atherosclerotic Occlusive Cerebrovascular Disease," NMC Case Report Journal, vol. 4, no. 1, pp. 27-32, 2017.

[13] K. Ogasawara, H. Yukawa, M. Kobayashi et al., "Prediction and monitoring of cerebral hyperperfusion after carotid endarterectomy by using single-photon emission computerized tomography scanning," Journal of Neurosurgery, vol. 99, no. 3, pp. 504510, 2003.

[14] M. Morioka, Y. Hasegawa, T. Kawano et al., "Prolonged and regionally progressive symptomatic cerebral hyperperfusion syndrome after superficial temporal artery-middle cerebral artery anastomosis in a patient with moyamoya disease," Surgical Neurology International, vol. 3, no. 1, p. 106, 2012.

[15] W. N. K. A. Van Mook, R. J. M. W. Rennenberg, G. W. Schurink et al., "Cerebral hyperperfusion syndrome," The Lancet Neurology, vol. 4, no. 12, pp. 877-888, 2005.

[16] S. Ivens, S. Gabriel, G. Greenberg, A. Friedman, and I. Shelef, "Blood-brain barrier breakdown as a novel mechanism underlying cerebral hyperperfusion syndrome," Journal of Neurology, vol. 257, no. 4, pp. 615-620, 2010.

[17] S. Oshida, K. Ogasawara, H. Saura et al., "Does preoperative measurement of cerebral blood flow with acetazolamide challenge in addition to preoperative measurement of cerebral blood flow at the resting state increase the predictive accuracy of development of cerebral hyperperfusion after carotid endarterectomy? Results from 500 cases with brain perfusion single-photon emission computed tomography study," Neurologia medico-chirurgica, vol. 55, no. 2, pp. 141-148, 2015.

[18] Y. Kaku, K. Iihara, N. Nakajima et al., "Cerebral blood flow and metabolism of hyperperfusion after cerebral revascularization in patients with moyamoya disease," Journal of Cerebral Blood Flow \& Metabolism, vol. 32, no. 11, pp. 2066-2075, 2012.

[19] O. Z. Chi, J. Grayson, S. Barsoum, X. Liu, A. Dinani, and H. R. Weiss, "Effects of dexmedetomidine on microregional $\mathrm{O} 2$ balance during reperfusion after focal cerebral ischemia," Journal of Stroke and Cerebrovascular Diseases, vol. 24, no. 1, pp. 163-170, 2015.
[20] H. Uchino, N. Nakayama, K. Kazumata, S. Kuroda, and K. Houkin, "Edaravone reduces hyperperfusion-related neurological deficits in adult moyamoya disease: Historical control study," Stroke, vol. 47, no. 7, pp. 1930-1932, 2016.

[21] M. Fujimura, K. Niizuma, T. Inoue et al., "Minocycline prevents focal neurological deterioration due to cerebral hyperperfusion after extracranial-intracranial bypass for moyamoya disease," Neurosurgery, vol. 74, no. 2, pp. 163-170, 2014.

[22] I. Udristoiu, I. Marinescu, MC. Pirlog et al., "The microvascular alterations in frontal cortex during treatment with antipsychotics: a post-mortem study," Romanian Journal of Morphology and Embryology, vol. 57, pp. 501-506, 2016.

[23] A. Gepdiremen, N. Aydin, Z. Halici et al., "Chronic treatment of haloperidol causes vasoconstriction on basilar arteries of rats, dose dependently," Pharmacological Research, vol. 50, no. 6, pp. 569-574, 2004.

[24] E. Elmorsy, L. M. Elzalabany, H. M. Elsheikha, and P. A. Smith, "Adverse effects of antipsychotics on micro-vascular endothelial cells of the human blood-brain barrier," Brain Research, vol. 1583, no. 1, pp. 255-268, 2014. 


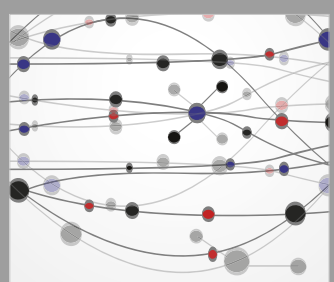

The Scientific World Journal
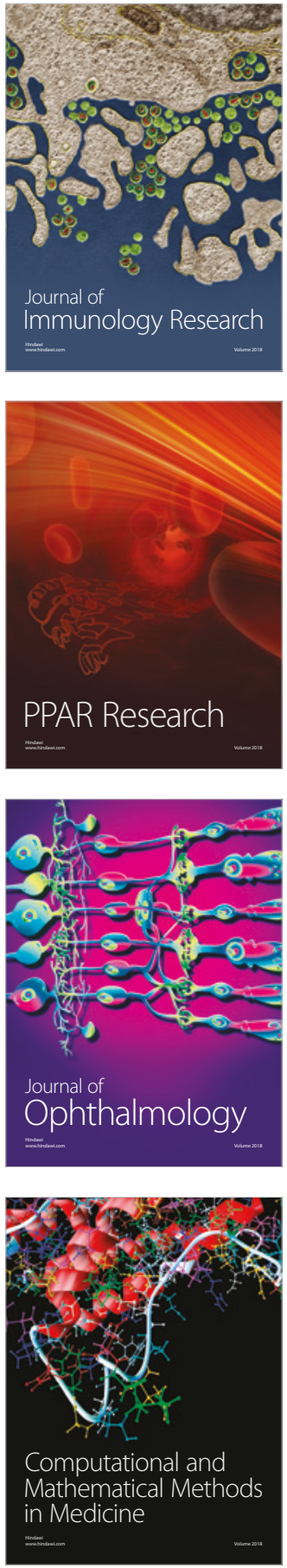

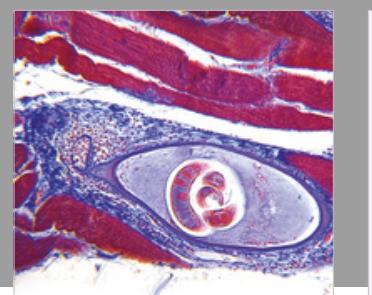

Gastroenterology Research and Practice

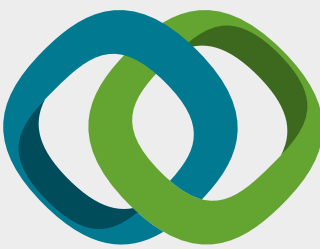

\section{Hindawi}

Submit your manuscripts at

www.hindawi.com
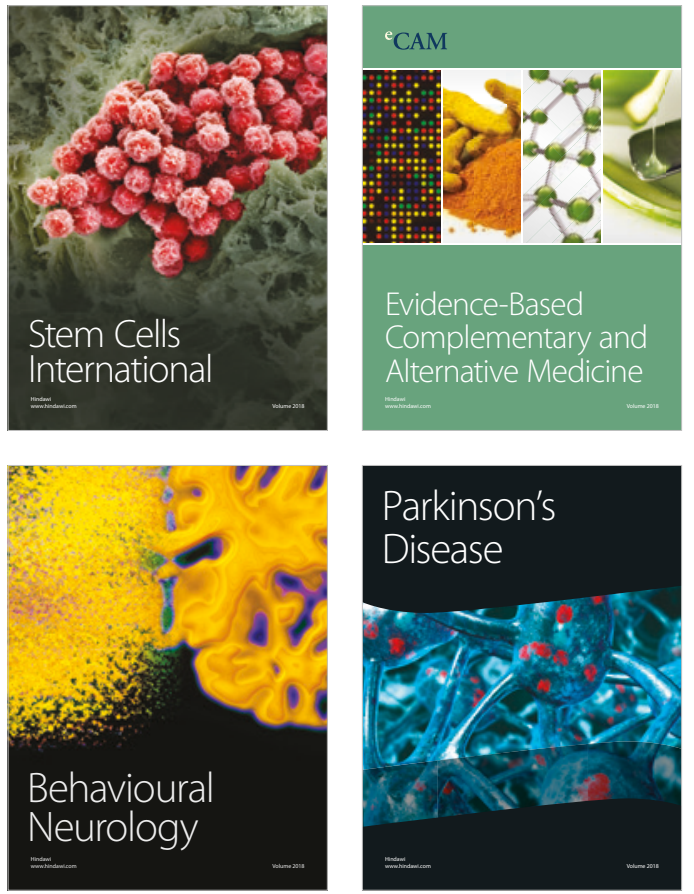

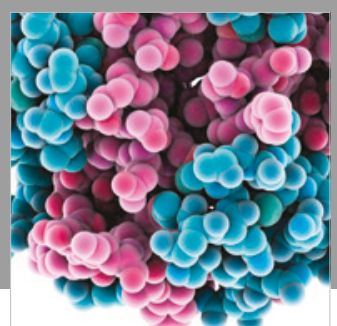

ournal of

Diabetes Research

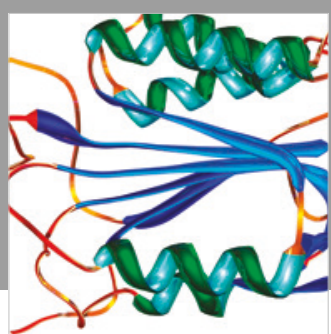

Disease Markers
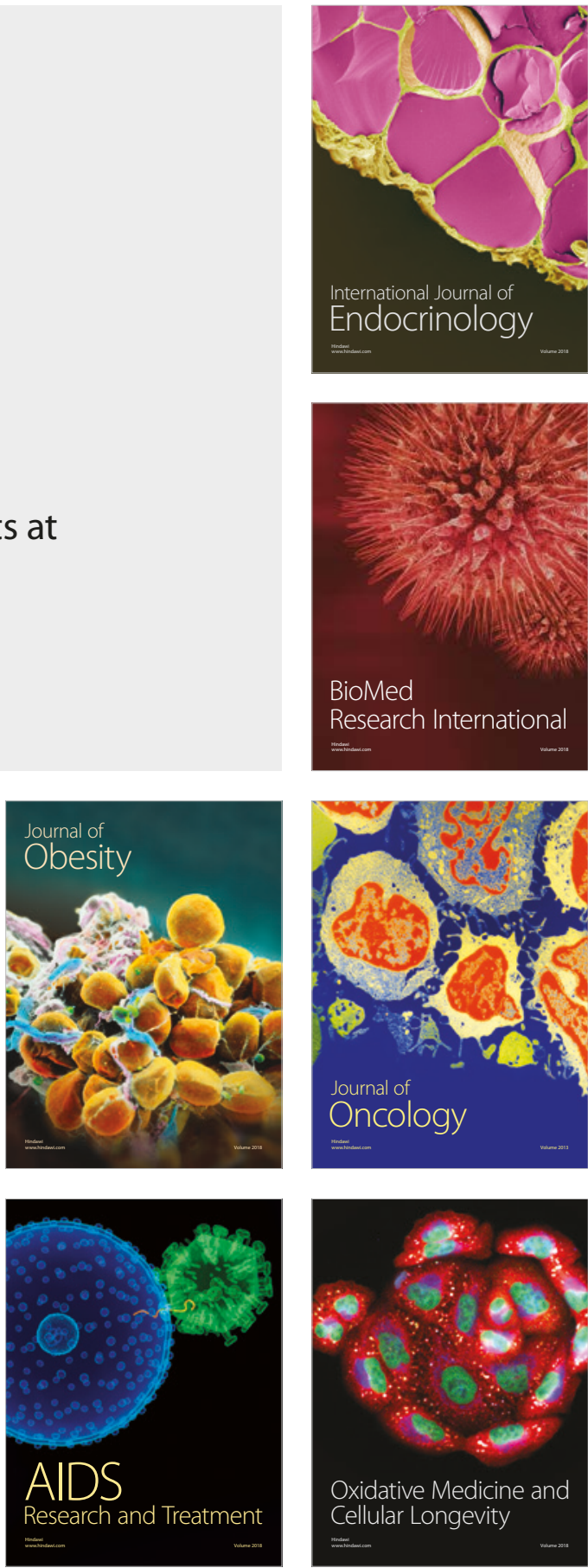